\title{
Contribution of the AIRS Shortwave Sounding Channels to Retrieval Accuracy
}

\author{
Joel Susskind $^{\mathrm{a}}$, Louis Kouvaris ${ }^{\mathrm{b}}$ \\ ${ }^{a}$ NASA Goddard Space Flight Center, Greenbelt, MD, USA 20771 \\ ${ }^{\mathrm{b}}$ SAIC, Beltsville, MD, USA, 20705
}

\begin{abstract}
AIRS contains 2376 high spectral resolution channels between $650 \mathrm{~cm}^{-1}$ and $2665 \mathrm{~cm}^{-1}$, including channels in both the 15 micron (near $667 \mathrm{~cm}^{-1}$ ) and 4.2 micron (near $2400 \mathrm{~cm}^{-1}$ ) $\mathrm{CO}_{2}$ sounding bands. Use of temperature sounding channels in the 15 micron $\mathrm{CO}_{2}$ band has considerable heritage in infra-red remote sensing. Channels in the 4.2 micron $\mathrm{CO}_{2}$ band have potential advantages for temperature sounding purposes because they are essentially insensitive to absorption by water vapor and ozone, and also have considerably sharper lower tropospheric temperature sounding weighting functions than do the 15 micron temperature sounding channels. Potential drawbacks with regard to use of 4.2 micron channels arise from effects on the observed radiances of solar radiation reflected by the surface and clouds, as well as effects of non-local thermodynamic equilibrium on shortwave observations during the day. These are of no practical consequences, however, when properly accounted for. We show results of experiments performed utilizing different spectral regions of AIRS, conducted with the AIRS Science Team candidate Version 5 algorithm. Experiments were performed using temperature sounding channels within the entire AIRS spectral coverage, within only the spectral region $650 \mathrm{~cm}^{-1}$ to $1614 \mathrm{~cm}^{-1}$; and within only the spectral region $1000 \mathrm{~cm}^{-1}-2665 \mathrm{~cm}^{-1}$. These show the relative importance of utilizing only 15 micron temperature sounding channels, only the 4.2 micron temperature sounding channels, and both, with regards to sounding accuracy. The spectral region $2380 \mathrm{~cm}^{-1}$ to $2400 \mathrm{~cm}^{-1}$ is shown to contribute significantly to improve sounding accuracy in the lower troposphere, both day and night.
\end{abstract}

Keywords: infra-red, microwave, remote sensing, temperature, moisture, high spectral resolution

\section{INTRODUCTION}

AIRS was launched on EOS Aqua on May 4, 2002, together with AMSU A and HSB, to form a next generation polar orbiting infrared and microwave atmospheric sounding system. ${ }^{1}$ The primary products of AIRS/AMSU are twice daily global fields of atmospheric temperature-humidity profiles, ozone profiles, sea/land surface skin temperature, and cloud related parameters including OLR. Also included are the clear column radiances used to derive these products which are representative of the radiances AIRS would have seen if there were no clouds in the field of view. All products also have error estimates. The sounding goals of AIRS are to produce $1 \mathrm{~km}$ tropospheric layer mean temperatures with an rms error of $1 \mathrm{~K}$, and layer precipitable water with an rms error of 20 percent, in cases with up to 80 percent effective cloud cover. The products are designed for data assimilation purposes for the improvement of numerical weather prediction, as well as for the study of climate and meteorological processes. With regard to data assimilation, one can use either the products themselves or the clear column radiances from which the products were derived.

The basic theory used to analyze AIRS/AMSU/HSB data in the presence of clouds, called the at-launch algorithm, and that used in a post-launch algorithm which differed only in the minor details from the at-launch algorithm, have been described previously ${ }^{2,3}$. The AIRS retrieval algorithm utilizes select subsets of the AIRS channels in the cloud clearing process, as well as in the physically based retrievals of different geophysical parameters. The temperature sounding physical retrieval step utilizes channels in both the $15 \mu \mathrm{m} \mathrm{CO}$ band and the $4.2 \mu \mathrm{m} \mathrm{CO}$ band. Channels in these two bands have different properties and are complimentary to each other. This results in improved sounding characteristics when channels in both spectral regions are used together compared to use of either separately. This improvement is even 
more significant when soundings are done in the presence of broken clouds (which occurs most of the time), because the cloud clearing process is more effective when channels in both spectral regions are used together.

In this paper, we briefly review the principles of cloud clearing and temperature sounding, and compare results of AIRS retrievals performed using selected subsets of all channels $\left(650 \mathrm{~cm}^{-1}-2665 \mathrm{~cm}^{-1}\right)$, only longwave temperature sounding channels $\left(650 \mathrm{~cm}^{-1}-1614 \mathrm{~cm}^{-1}\right)$, and only shortwave temperature shortwave channels $\left(1000 \mathrm{~cm}^{-1}-2665 \mathrm{~cm}^{-1}\right)$. These results include having the benefit of colocated AMSU-A observations. The considerations in this paper are also relevant to the design of future high spectral resolution IR instruments, such as HES which will fly on the geostationary satellite GOES-R in the future. GOES-R will not (at least in its earliest years) have the benefit of a colocated microwave geostationary sounder. Therefore analogous results are also shown which do not include microwave channels in the retrieval or quality control process.

Susskind $^{4}$ shows recent results of analysis of AIRS/AMSU data with an improved retrieval algorithm, referred to as AIRS candidate Version 5.0. All experiments done in this paper use the basic formalism of AIRS candidate Version 5.0, with the exception of the new candidate Version 5.0 quality control methodology.

\section{OVERVIEW OF THE AIRS TEAM RETRIEVAL ALGORITHM}

The AIRS team candidate Version 5.0 retrieval algorithm is basically identical to that described in Susskind et al. ${ }^{2,3 .}$ The key steps of the AIRS candidate Version 5.0 algorithm are outlined below: 1) Start with an initial state, called the MW retrieval state, consistent with the AMSU A and HSB radiances ${ }^{5}$; 2) Derive IR clear column radiances $\hat{R}_{i}^{0}$ valid for the $3 \times 3$ AIRS Fields of View (FOVs) within an AMSU A Field of Regard (FOR) consistent with the observed radiances and the initial state; 3) Obtain an AIRS regression guess ${ }^{6}$ consistent with $\hat{R}_{i}^{0}$ using a subset of AIRS channels; 4) Derive $\hat{R}_{i}^{1}$ consistent with the AIRS (and AMSU) radiances and the regression guess; 5) Derive all surface and atmospheric parameters using $\hat{R}_{\mathrm{i}}^{1}$ for a subset of AIRS channels (and AMSU) radiances; 6) Derive an improved set of clear column radiances $\hat{R}_{i}^{2}$ using the AIRS physically retrieved parameters; 7) Repeat Step 5 using $\hat{R}_{i}^{2}$ to produce the final IR/MW retrieval state; 8) Apply initial quality control, which rejects a IR/MW solution if the retrieved cloud fraction is greater than $90 \%$ or other relatively coarse tests fail. Further quality control is then applied to individual geophysical parameters. This quality control procedure is different in Version 4.0 and candidate Version 5.0. We have not yet derived the necessary Version 5.0 quality control matrices for each of the experiments done. Therefore, Version 4.0 type quality control was used for this paper.

\section{BASIC THEORETICAL CONSIDERATIONS WITH REGARD TO ATMOSPHERIC SOUNDING}

The cloud free radiance going to space for channel $\mathrm{i}, \mathrm{R}_{\mathrm{i}}$, assuming Local Thermodynamic Equilibrium, is approximately given by

$$
R_{i}=\varepsilon_{i} B_{v_{i}}\left(T_{s}\right) \tau_{i}\left(p_{s}\right)+\int_{P_{s}}^{0} B_{v_{i}}[T(p)] W_{i}(p) d \ell n p+S_{i}
$$

where $\tau_{i}(p)$ is the atmospheric transmittance from pressure $p$ to the satellite, $B_{v_{i}}(T)$ is the Planck Black Body function, $\mathrm{W}_{\mathrm{i}}(\mathrm{p})=\mathrm{d} \tau_{\mathrm{i}} / \mathrm{d} \ell \mathrm{np}, \varepsilon_{\mathrm{i}}$ is the surface spectral emissivity at frequency $v_{\mathrm{i}}$, and $\mathrm{S}_{\mathrm{i}}$ reflects solar radiation transmitted to the surface, reflected and transmitted back to the satellite. $\tau_{\mathrm{i}}(\mathrm{p})$ can vary from 1 , representing total transmission by the atmosphere to the satellite, to 0 , representing total atmospheric opacity at that pressure. The first term represents radiation emitted by the surface and transmitted to the satellite, and the second term represents radiation emitted by the 
atmosphere and transmitted to the satellite. Ignoring the last term, and assuming unit surface emissivity, the radiance can be approximated as a linear combination of radiance coming from the surface and the atmosphere

$$
\mathrm{R}_{\mathrm{i}} \approx \tau_{\mathrm{i}}\left(\mathrm{p}_{\mathrm{s}}\right) \mathrm{B}\left(\mathrm{T}_{\mathrm{s}}\right)+\left[1-\tau_{\mathrm{i}}\left(\mathrm{p}_{\mathrm{s}}\right)\right] \mathrm{B}_{\mathrm{i}}(\mathrm{T}(\overline{\mathrm{p}}))
$$

where $T\left(\bar{p}_{i}\right)$ is the temperature at an effective pressure $\bar{p}$, within the pressure region in which $\tau_{i}(p)$ varies about $\mathrm{e}^{-1}$. The sharper $W_{i}(p)$, referred to as the temperature weighting function, the narrower the pressure region corresponding to atmospheric emission. It is convenient to write $R_{i}$ in brightness temperature units, $\Theta_{i}$, in which $\Theta_{i}=B_{v_{i}}^{-1}\left(R_{i}\right)$. The channel brightness temperature can then be approximated as

$$
\Theta_{\mathrm{i}}=\tau\left(\mathrm{p}_{\mathrm{s}}\right) \mathrm{T}_{\mathrm{s}}+\left(1-\tau\left(\mathrm{p}_{\mathrm{s}}\right)\right) \mathrm{T}\left(\overline{\mathrm{p}}_{\mathrm{i}}\right)
$$

For channels in which there is little atmospheric absorption, $\tau_{i}\left(p_{s}\right) \approx 1.0$ and $\Theta_{i} \approx T_{s}$. For opaque channels, $\Theta_{i} \approx T\left(\bar{p}_{i}\right)$. The stronger the absorption, the lower the value of $\bar{p}_{i}$, and $T\left(\bar{p}_{i}\right)$ decreases with decreasing $\bar{p}_{i}$ (increasing absorption) in the troposphere, with the reverse being true for $\bar{p}_{i}$ in the stratosphere. Atmospheric absorption in the infrared is due primarily to absorption by $\mathrm{CO}_{2}$, with absorption features centered near $667 \mathrm{~cm}^{-1}$ and $2350 \mathrm{~cm}^{-1}, \mathrm{H}_{2} \mathrm{O}$, centered near $1600 \mathrm{~cm}^{-1}$; and $\mathrm{O}_{3}$, centered near $1040 \mathrm{~cm}^{-1}$.

For temperature sounding purposes, ideally one would like to have channels in which the concentration of the absorbing species is well known $\left(\mathrm{CO}_{2}\right)$, the channel weighting functions are very narrow, and there is little contamination by additional absorption due to gasses with variable concentrations such as $\mathrm{H}_{2} \mathrm{O}$ and $\mathrm{O}_{3}$. One would also like to have sensitivity to temperature at all levels of the atmosphere. For the purpose of determining the vertical distribution of gasses with variable mixing ratios, one chooses channels primarily sensitive to absorption and emission by the gas in question.

Figure 1 shows an AIRS brightness temperature spectrum. Also indicated in the figure are those AIRS channels used in candidate Version 5.0 for different purposes in the physical retrieval process. Individual $\mathrm{CO}_{2}$ absorption lines are evident in the spectral region $650 \mathrm{~cm}^{-1}-750 \mathrm{~cm}^{-1}$. For channels with characteristic pressures $\overline{\mathrm{p}}_{i}$ in the troposphere, the peak absorption of the line is a local minimum in the brightness temperature spectrum, while for channels with $\overline{\mathrm{p}}_{\mathrm{i}}$ in the stratosphere, channel centers appear as a local maximum. The strong brightness temperature maximum near $666 \mathrm{~cm}^{-1}$ (the $\mathrm{CO}_{2}$ Q-branch) is the most opaque region of the spectrum and is representative of atmospheric emission near $1 \mathrm{mb}$. Individual spectral features are also evident in the water vapor and ozone absorption regions. Essentially no spectral structure is seen in the $\mathrm{CO}_{2}$ absorption region in the vicinity of $2350 \mathrm{~cm}^{-1}$ however, because the $\mathrm{CO}_{2}$ line spacing is less than the spectral resolution of AIRS, which degrades with increasing wave number.

\subsection{Properties of temperature sounding channels in different spectral regions}

Historically, channels in the $15 \mu \mathrm{m} \mathrm{CO}$ spectral band have the greatest heritage for use in temperature sounding purposes. These channels have good sensitivity to atmospheric temperatures ranging from the surface (near $750 \mathrm{~cm}^{-1}$ ) to $1 \mathrm{mb}$ (near $666 \mathrm{~cm}^{-1}$ ). Kaplan et al. ${ }^{7}$ show that the sharp temperature weighting functions occur between $\mathrm{CO}_{2}$ absorption lines, which are well resolved by AIRS. A complication to use of the $15 \mu \mathrm{m}$ band for temperature sounding results from the effects of absorption by water vapor and ozone lines in that spectral region. Water vapor absorption is especially significant for those channels sensitive to mid-lower tropospheric emission. Sounding channels in the 4.3 micron $\mathrm{CO}_{2}$ spectral band have quite different characteristics. This spectral region has very little absorption by water vapor or ozone. More significantly, Kaplan et al. ${ }^{7}$ show that the sharpest mid-lower tropospheric temperature weighting functions are 
found in the spectral region $2380 \mathrm{~cm}^{-1}-2392 \mathrm{~cm}^{-1}$. On the other hand, because of the large temperature dependence of the Planck Black Body function near $2400 \mathrm{~cm}^{-1}$, channel radiances in this region are less sensitive to temperatures in the cold tropopause region than those near $660 \mathrm{~cm}^{-1}$. Further complications with regard to use of these shortwave channels are the significant effects of the term $S_{i}$ (reflected solar radiation) on shortwave channels sensitive to the surface skin and lower tropospheric emission, and of non Local Thermodynamic Equilibrium (non-LTE) on shortwave channels sensitive to the upper troposphere and stratosphere. Both of these factors, occurring only under sun-up conditions, have discouraged researchers from using these channels for sounding (or data assimilation) purposes, especially during the day.

Another set of temperature sounding channels, in the $56 \mathrm{GHz}, \mathrm{O}_{2}$ band, are found on the microwave sounder AMSU-A. These channels have sharp upper tropospheric and stratospheric temperature sounding channels, and good vertical coverage of the atmosphere to the surface. The weighting functions for channels sensitive to the mid-lower troposphere are relative broad however, and moreover, these channels are also affected by uncertainties in the surface spectral emissivity.
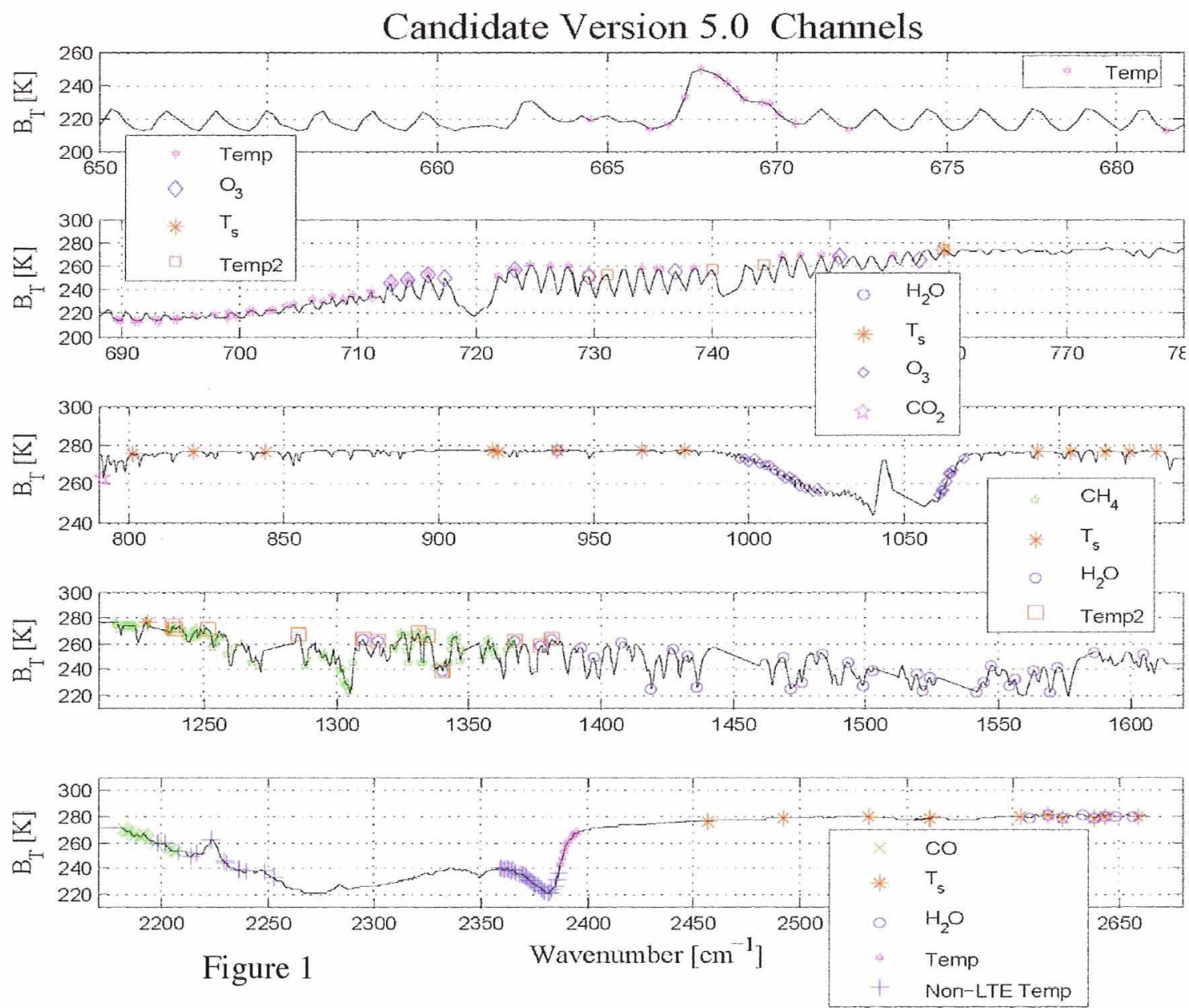


\subsection{Use of temperature sounding channels in candidate Version $\mathbf{5 . 0}$}

Candidate Version 5.0 uses all good AIRS and AMSU channels in different ways. All good AMSU channels are used to obtain a start-up state needed for initial cloud clearing (Step 1). This is used to get $R_{i}^{0}$ (Step 2). In addition, a temperature profile retrieval using only AMSU channels is performed after the AIRS regression step (Step 3). This modified state, consistent with AIRS and AMSU radiances, is used to derive $\hat{R}_{i}{ }^{2}$ (Step 4). AMSU channels are not used subsequently except for quality control purposes. All good AIRS channels in the spectral range $650 \mathrm{~cm}^{-1}-2665 \mathrm{~cm}^{-1}$ are used in the regression step (Step 3). The physical retrieval steps use only the subsets of channels shown in Figure 1. Temperature sounding channels not subject to non-LTE effects are shown in red. Most are in the $15 \mu \mathrm{m} \mathrm{CO}_{2}$ band, inbetween $\mathrm{CO}_{2}$ absorption lines. $\mathrm{CO}_{2}$ channels highly sensitive to water vapor or ozone absorption are not used. Also used are channels on the $666 \mathrm{~cm}^{-1} \mathrm{CO}_{2}$ Q-branch that contain sensitivity to temperatures up to $1 \mathrm{mb}$. Extensive use is made of the temperature sounding channels in the spectral region $2350 \mathrm{~cm}^{-1}-2392 \mathrm{~cm}^{-1}$. Most of the shortwave channels, shown in blue, are sensitive to effects of non-LTE, and the remainder shown in red, are sensitive to solar radiation reflected by the surface (and clouds). These factors are well accounted for in the physics used in the candidate Version 5.0 methodology and these channels are used equally well during the day and night.

\section{BASIC THEORETICAL CONSIDERATIONS WITH REGARD TO CLOUD CLEARING}

Using the assumption that adjacent fields of view have otherwise identical geophysical conditions except for cloud cover, Chahine ${ }^{8}$ has shown that in the case of K-1 cloud formations, observations in K fields of view (FOV's) are needed to obtain channel i clear column radiances $\hat{\mathrm{R}}_{\mathrm{i}}$ according to

$$
\hat{R}_{i}=R_{i, 1}+\sum_{k=1}^{K-1} \eta_{k}\left(R_{i, 1}-R_{i, K+1-k}\right)
$$

where $R_{i, k}$ is the channel $i$ observation in field of view $k$. The parameters $\eta_{k}$ should be valid for all channels. In analyzing AIRS/AMSU data, we produce soundings using all 9 AIRS FOV's falling within a single AMSU A footprint. We have found it is advantageous to extrapolate the radiances in the 9 fields of view according to a similar equation of the form

$$
\hat{R}_{i}=R_{i, A V G}+\sum_{k=1}^{8} \eta_{k}\left(R_{i, A V G}-R_{i, k}\right)
$$

where $R_{i, A V G}$ is the average radiance of all 9 fields of view. Optimal values of $\eta_{k}$ will give true values of $\hat{R}_{j}$ up to instrumental noise effects. Given values of $\eta_{k}, \hat{R}_{i}$ is determined using equation 5 .

$\eta_{\mathrm{k}}$ is determined using an ensemble of I cloud filtering channels with temperature weighting functions covering the atmosphere from the lower stratosphere to the surface, as well a some window channels. If, for each channel $i$, one substitutes an estimate of $R_{i, C L R}$ for $\hat{R}_{i}$ in Equation 5, this gives I equations for $K(=8)$ unknowns. The unconstrained weighted least square solution to this multilinear problem is given by

$$
\eta_{K x 1}=\left[\Delta R^{\prime} N^{-1} \Delta R\right]_{K x K}^{-1} \Delta R^{\prime} N^{-1} \Delta R_{C L R}
$$


where $\Delta R$ is a IxK matrix with $\Delta R_{i, k}=R_{A V G}-R_{i, k}, \Delta R_{C l R}$ is an IxI matrix given by $\Delta R_{i, C L R}=R_{i, C L R}-R_{i, A V G}$, and $\mathrm{N}$ is an IxI channel noise covariance matrix.

The key to the accurate determination of $\eta$ is obtaining the best estimates of $\Delta \mathrm{R}_{\mathrm{i}, \mathrm{CLR}}$, along with an accurate treatment of the noise covariance matrix $N$. The values of $\triangle R_{i, C L R}$ which we use to determine $\eta$ (and $\hat{R}_{i}$ ) are iterative and are computed based on the current best estimate of all relevant surface and atmospheric properties. For optimal results, it is important for the estimated geophysical parameters to be unbiased over large regions of the atmosphere. For example, if the estimated temperature profile were uniformly too warm, values of $\Delta \mathrm{R}_{\mathrm{i}, \mathrm{CLR}}$ would all be too high and incorrect values of $\eta_{k}$ would be obtained which would reconstruct too high values of $\hat{R}_{i}$. To avoid this, we make sure that the profile used to estimate $R_{i, C L R}$ is consistent with observations in all AMSU A and HSB channels, thus insuring an unbiased temperature and moisture profile over coarse layers in the atmosphere. $\hat{R}_{i}^{0}$ uses the initial profile derived from the AMSU only retrieval to compute $\mathrm{R}_{\mathrm{i}, \mathrm{CLR}}$, and $\hat{\mathrm{R}}_{\mathrm{i}}{ }^{1}$ uses the geophysical state obtained by following the AIRS regression with another AMSU retrieval.

If the state used to derive $R_{i, C L R}$ were biased (say too warm), incorrect values of $\eta_{k}$ would be determined which would result in $\hat{R}_{i}$ being too large, which in turn would result in the retrieved $T(p)$ being too warm. Chahine has shown that it is optimal to use only longwave ( $15 \mu \mathrm{m}$ and $12 \mu \mathrm{m}$ ) channels for cloud clearing, and shortwave channels for sounding temperatures in the mid-lower troposphere. This is done so as to minimize the bias in retrieved temperature profiles resulting from biases in the temperature profile used to determine $\eta_{\mathrm{k}}$.

\section{AIRS/AMSU RETRIEVALS}

AIRS/AMSU retrievals were run on a global data set for September 6, 2002. Results are shown for experiments using all AIRS channels (LW/SW), analyzed using a small modification of the candidate Version 5.0 AIRS retrieval system ${ }^{3}$, with Version 4.0 quality control ${ }^{2}$. Also shown are results using only the subset of AIRS channels $650 \mathrm{~cm}^{-1}-1614 \mathrm{~cm}^{-1}$ (LW), and $1000 \mathrm{~cm}^{-1}-2665 \mathrm{~cm}^{-1}$ (SW). Separate AIRS regression coefficients were generated for each of the three experiments, using only the subset of AIRS channels present in the experiment. The LW/SW experiment used LW AIRS channels for cloud clearing and temperature sounding in the mid-troposphere through the stratosphere, and SW channels for temperature sounding through the entire atmosphere. The LW experiment did not have the benefit of SW temperature sounding channels. Therefore LW temperature sounding channels were used to sound the mid-lower troposphere as well. The SW experiment had neither the benefit of $\mathrm{LW}$ temperature sounding channels or the LW window region from $750 \mathrm{~cm}^{-1}-1000 \mathrm{~cm}^{-1}$. Therefore, $\mathrm{SW}$ temperature sounding and window channels were used for cloud clearing. In addition, more SW window channels were used in the surface parameter physical retrieval step than in the LW/SW system. The water vapor sounding spectral region is common in all experiments and no changes were made to the channels used in the water vapor physical retrieval step.

\subsection{Results}

Figure 2 shows RMS differences from "truth" (taken as the colocated ECMWF 3-hour forecast), for $1 \mathrm{~km}$ thick layer mean temperatures, for a global ensemble of accepted retrievals, for each of the three experiments described. The physical retrieval solutions are shown in solid lines, and the first guess, obtained by following the AIRS regression step by an AMSU only physical retrieval, are shown in dashed lines. The accuracies of all first guesses are similar above 160 $\mathrm{mb}$, as a result of the ability of the AMSU retrieval step to correct regression errors in the stratosphere. Beneath $160 \mathrm{mb}$, the LW/SW and LW first guesses are again almost identical, but the SW first guess is less accurate. This is a result of factors arising from two different considerations. First, linear regression performs much better using LW channels than $S W$ channels because $L W$ radiances are much more linear with increasing temperature than SW radiances. In addition, the Planck Black Body function $B_{v}(T)$ is much more linear in $T$ in the $L W$ than in the $S W$. SW radiances are also affected by solar radiation and non-LTE effects. Hence, regression results using LW and LW/SW channels are very 
similar to each other, while regression using SW radiances alone performs poorer. Secondly, microwave observations contain fewer and coarser temperature weighting functions than IR observations in the mid-lower troposphere and therefore cannot improve significantly upon the SW only regression. The accuracies of LW/SW and LW only AIRS/AMSU physical temperature retrievals are almost identical down to the mid-troposphere $(\approx 500 \mathrm{mb})$, but the LW/SW system is considerably more accurate deeper in the atmosphere. Part of this improvement is due to the benefit of using $\hat{\mathrm{R}}_{\mathrm{i}}{ }^{2}$ for the final retrieval, while $\hat{\mathrm{R}}_{\mathrm{i}}{ }^{0}$ is used to generate the regression, and part is due to the enhanced vertical resolution of the SW temperature sounding channels between $2380 \mathrm{~cm}^{-1}$ and $2392 \mathrm{~cm}^{-1}$. It is noteworthy that both the LW/SW and SW only physical retrievals improve significantly upon their first guess in the mid-lower troposphere, while the $L W$ only physical retrieval does not. In the $L W$ case, the benefit of improved values of $\eta^{2}$ is mitigated with regard to improving $\hat{R}_{i}{ }^{2}$ by the fact that $L W$ channels are used for both cloud clearing and mid-lower tropospheric temperature sounding. Hence, the LW physical retrieval does not improve over the first guess in the lower troposphere.

Figure 2 contains results for both day and nighttime cases. Figure 3 shows analogous results for daytime cases only, in which the shortwave window and temperature sounding channels are affected by solar radiation reflected by clouds and the surface, and non-LTE effects are present as well. General findings during the day with regard to the three first guesses and physical retrievals are basically similar to those found day and night together, though the improvement of the LW/SW system is slightly less than during the day relative to the LW only system. A degradation in the lower troposphere occurs in the LW/SW first guess compared to the LW first guess during the day. The LW/SW physical retrieval improves on this result, while the LW only physical retrieval is again unable to improve on its first guess. This minor degradation in LW/SW results during the day show that the effects of solar radiation reflected by clouds on SW observations is well accounted for by the cloud clearing procedure, and the effect of solar radiation reflected by the surface and non-LTE effects are well accounted for in the retrieval physics.

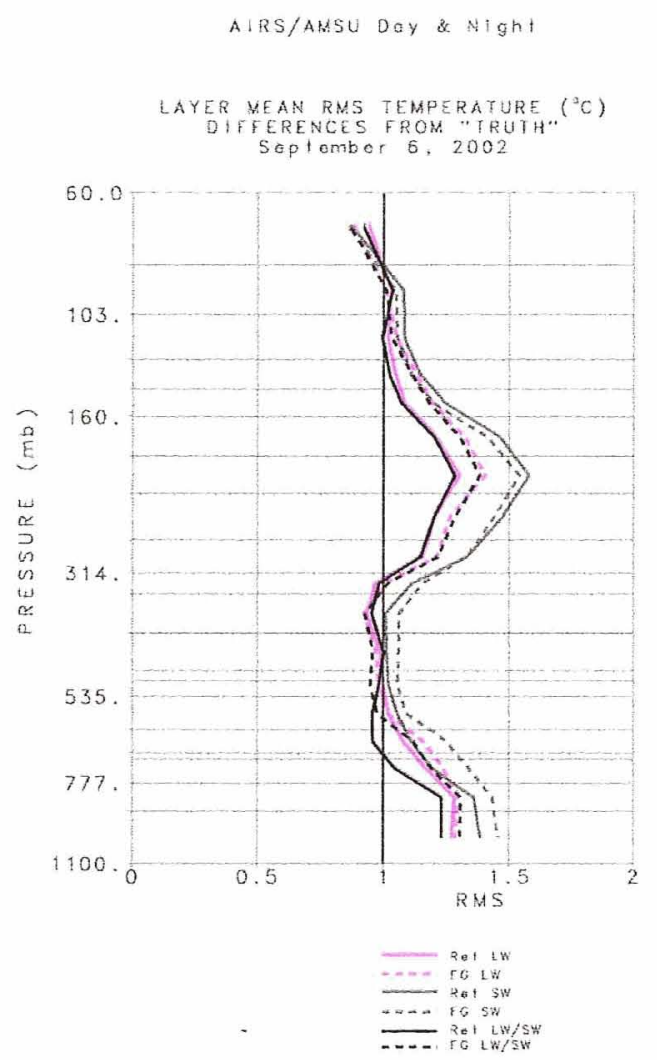

Figure 2

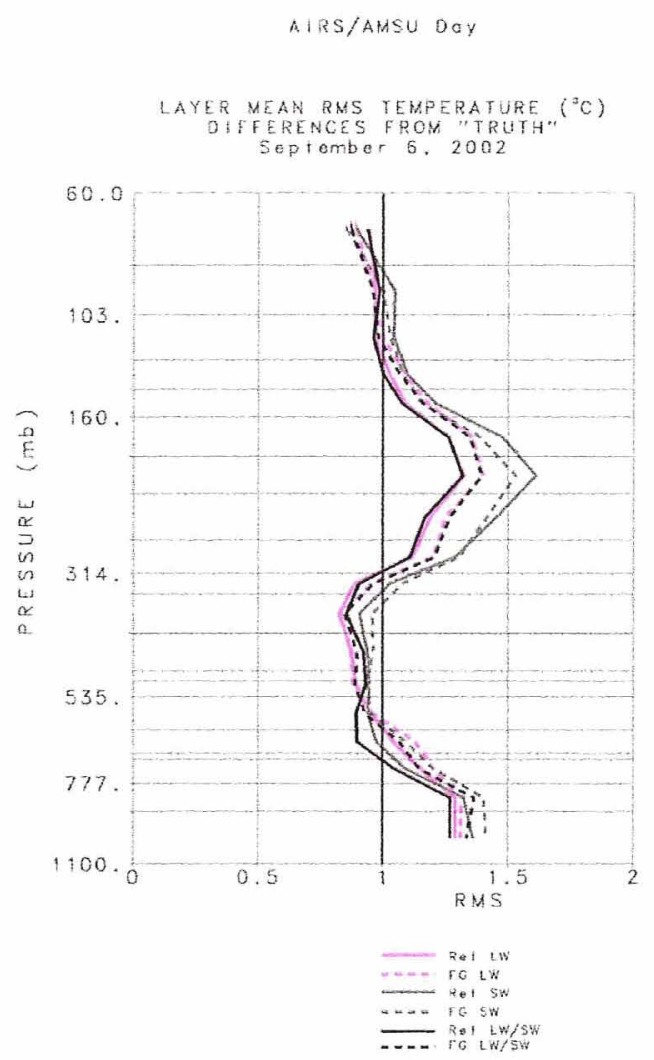

Figure 3 


\subsection{AIRS ONLY RETRIEVALS}

In an AIRS/AMSU system, we have demonstrated that a LW/SW sounding system performs better than a LW or a SW sounding system by itself, both day and night. The improvement is in the mid-lower troposphere, where shortwave temperature sounding channels provide the best information about the lower troposphere, especially when longwave channels are used to generate the cloud cleared radiances. Results of all three experiments were similar to each other in the stratosphere, as a result of information provided by AMSU A. The shortwave only system performs poorest in the tropopause region near $200 \mathrm{mb}$, where cold temperatures limit the signal coming from radiances near 4.2 microns.

The AIRS only set of experiments was oriented towards considerations related to the design of the HES high-spectral resolution sounder scheduled to fly on GEOS-R, which will not contain a microwave sounding instrument. These experiments were conducted exactly as the AIRS/AMSU set of experiments, but AMSU observations were not used in the retrieval process other than to provide the state used to determine the initial cloud cleared radiances $\hat{R}_{i}^{0}$. An AMSU retrieval was not performed after the IR regression state, and no AMSU channel observations were included in any physical retrieval steps or in the quality control. In reality, one would most likely use a GCM forecast state to determine $\hat{\mathrm{R}}_{\mathrm{i}}{ }^{0}$, which would have the benefit, via data assimilation, of AMSU observations from polar satellites.

In an attempt to simplify the design (and reduce the potential cost) of the HES sounder, the HES spectral coverage will most likely be truncated from that of AIRS to lie between $680 \mathrm{~cm}^{-1}$ and $2400 \mathrm{~cm}^{-1}$. Therefore, the AIRS only runs were done in the following spectral ranges: LW/SW $680 \mathrm{~cm}^{-1}-2400 \mathrm{~cm}^{-1}$; LW $680 \mathrm{~cm}^{-1}-1614 \mathrm{~cm}^{-1}$; and SW $1000 \mathrm{~cm}^{-1}-$ $2400 \mathrm{~cm}^{-1}$. The spectral region $650 \mathrm{~cm}^{-1}-680 \mathrm{~cm}^{-1}$ contains information about mid-upper stratospheric temperatures. This would have been useful to have on HES, but is not critical because sounding goals from Geostationary satellite are more oriented toward accuracy in the troposphere and lower stratosphere.

\subsection{Results}

Figures 4 and 5 show analogous results to those of Figures 2 and 3, but for the AIRS only retrievals. In these figures, the first guess shown is representative of the AIRS regression state itself, which does not have the benefit of being corrected by AMSU observations. All findings of the AIRS/AMSU retrieval experiments hold with regard to AIRS only retrievals, but to even a larger degree because AMSU is not present to help correct regression errors in the mid-lower troposphere. The first guess has similar accuracy in the stratosphere in the LW/SW and LW systems, because most of the information is coming from the $\mathrm{LW} \mathrm{CO}$ band. The accuracy of the $\mathrm{SW}$ regression first guess is much poorer in the upper troposphere and stratosphere, above $300 \mathrm{mb}$. Figure 4 (day and night) shows the upper tropospheric first guess is significantly better using the LW/SW system than LW alone. This is a result of degraded cloud cleared radiances $\hat{\mathrm{R}}_{\mathrm{i}}{ }^{0}$ obtained in the LW only system compared to the LW/SW system. The regression first guess of all three systems are poorer in the lower troposphere, day and night, than those found when the AMSU retrieval was used to adjust the regression state. This is primarily a result of the AMSU temperature retrieval step being able to partially correct for effects of cloud clearing errors on $\hat{\mathrm{R}}_{\mathrm{i}}{ }^{0}$, which is used to generate the regression state. These cloud clearing errors in $\hat{R}_{i}{ }^{0}$ are largest in the SW system, where one can only use shortwave channels in the cloud clearing process. In the HES scenario, results in the lower troposphere are significantly better with a combined LW/SW sounder compared to a $\mathrm{LW}$ only sounding system. As with AIRS/AMSU, this is a result both of the enhanced vertical resolution in the lower troposphere found in the shortwave $\mathrm{CO}_{2}$ band, and even more so, the enhanced ability to correct for cloud clearing errors when shortwave channels are used in the retrieval process and longwave channels used for cloud clearing. As in the AIRS/AMSU retrievals, the LW physical retrieval is less able to improve on the regression, while the LW/SW and SW physical retrievals improve significantly over the regression guess.

These experiments indicate it would be optimal for HES to contain spectral coverage in both the $\mathrm{LW}$ and $\mathrm{SW}_{2} \mathrm{CO}_{2}$ bands. If, for technical (cost) reasons, one had to choose only the longwave or shortwave $\mathrm{CO}_{2}$ bands, results in the lower troposphere, while degraded, are roughly comparable to each other with either subset of channels. 


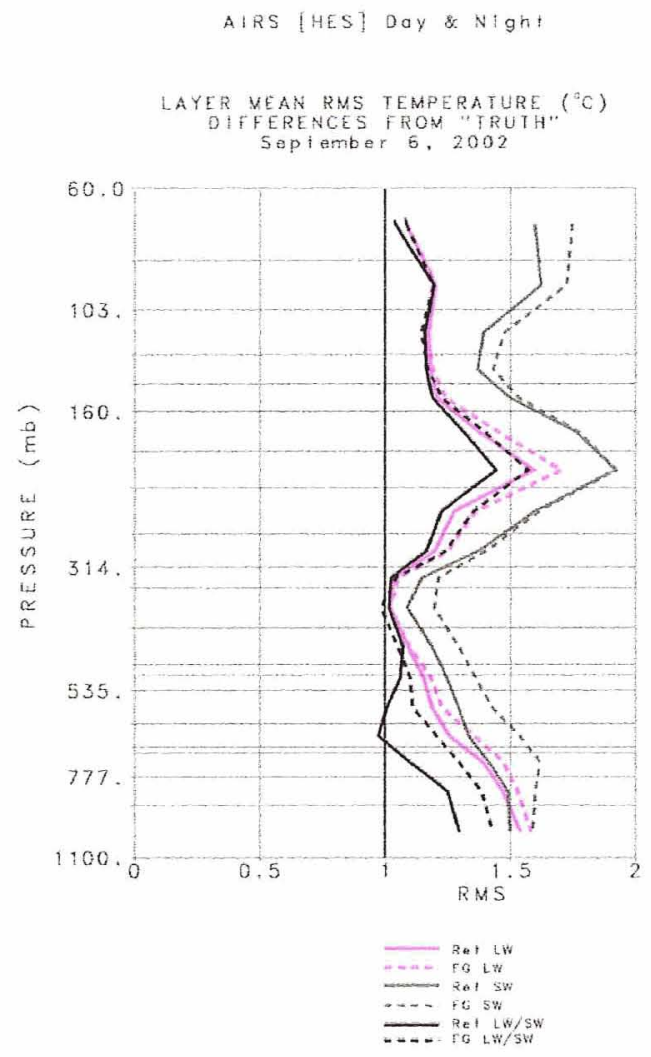

Figure 4

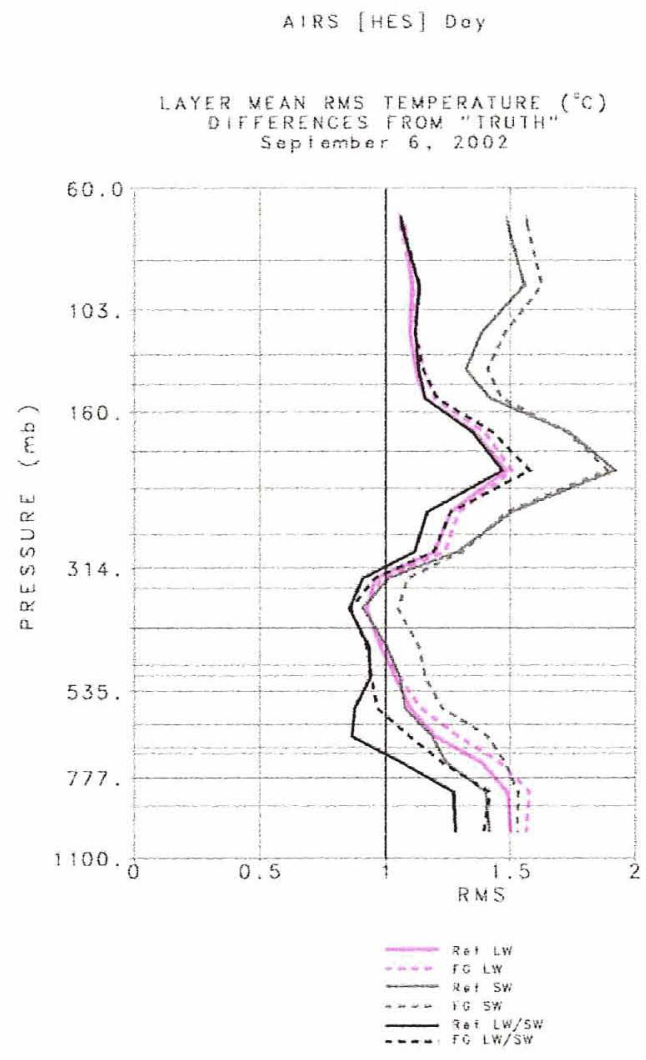

Figure 5

\section{REFERENCES}

1. Pagano, T. S., H. H. Aumann, D. E. Hagan, and K. Overoye, Prelaunch and in-flight radiometric calibration of the Atmospheric Infrared Sounder (AIRS). IEEE Trans. Geosci. Remote Sensing, 41, 265-273, February 2003.

2. Susskind, J., C. D. Barnet, and J. M. Blaisdell, Retrieval of atmospheric and surface parameters from AIRS/AMSU/HSB data in the presence of clouds. IEEE Trans. Geosci. Remote Sensing, 41, 390-409, February 2003.

3. Susskind, J., C. Barnet, J. Blaisdell, L. Iredell, F. Keita, L. Kouvaris, G. Molnar, and M. Chahine, Accuracy of geophysical parameters derived from AIRS/AMSU as a function of fractional cloud cover. J. Geophys. Res., 2006, in press.

4. Susskind, J., Improved soundings and error estimates using AIRS/AMSU. Proc. of SPIE, Vol. 6233, 2006.

5. Rosenkranz, P. W., Retrieval of temperature and moisture profiles from AMSU-A and AMSU-B measurements. In Proc. IGARSS, 2000. 
6. Goldberg, M. D., Y. Qu, L. M. McMillin, W. Wolff, L. Zhou, and M. Divakarla, AIRS near-real-time products and algorithms in support of operational numerical weather prediction. IEEE Trans. Geosci. Remote Sensing, 41, 379-389, February 2003.

7. Kaplan, L. D., M. T. Chahine, J. Susskind, and J. E. Searl, Spectral band passes for a high precision satellite sounder. Appl. Opt., 16, 322-325, 1977.

8. Chahine, M. T., Remote sensing of cloudy atmospheres. II. Multiple cloud formations. J. Atmos. Sci., 34, 744-757, 1977.

9. Chahine, M. T., Remote sensing of cloud atmospheres. I. The single cloud layer. J. Atmos. Sci., 31, 233-243, 1974. 